\title{
CORRECTIONS
}

\section{Afghanistan: a healthy future?}

In this Feature by Kate Adams (BMJ 2014;348:g3950, doi:10. 1136/bmj.g3950), we misspelt Malalai Hospital as "Malalay

Hospital" and incorrectly stated that it is run by Première

Cite this as: BMJ 2014;348:g4093

Urgence-Aide Médicale Internationale, when it is, in fact, 